\title{
A Good Chance for the Cat's Life: Erwin Schroedinger's New Statistics and a Second Quantization Theory of Quantum
}

\author{
Salvo D'Agostino \\ University of Roma "Sapienza", Rome, Italy \\ Email: saldagostino21@gmail.com
}

Received 19 December 2014; accepted 6 January 2015; published 30 March 2015

Copyright (C) 2015 by author and Scientific Research Publishing Inc.

This work is licensed under the Creative Commons Attribution International License (CC BY). http://creativecommons.org/licenses/by/4.0/

c) (i) Open Access

\begin{abstract}
In his paper on the Cat's uncertain situation between life and death, $S$. intended to criticize the Copenhagen interpretation of Quantum Mechanics and, conversely, to assert the consistency of his own wavelike view of the theory. His ideas contrasted with Heisenberg and Bohr's positions, but also with Einstein's attempt at a classically statistical interpretation of a quantum theory. His criticism therefore did not intend to discredit the whole Quantum Mechanics. In the fifties, he proposed a second quantization approach to a many particles state of the theory, which included his New Statistics. He asserted that the new approach modified some aspects of his famous 1926 equations. In my paper, I argue that S.'s new ideas in the fifties are related to his 1920 and 1926 contributions on rarified gas theory.
\end{abstract}

Keywords

Schroedinger, The Cat, Second Quantization Theory

\section{Introduction}

Erwin Schrödinger’s (1887-1961) presented his famous Cat episode in his 1935 essay “Die Gegenwärtige Situation in der Quantenmechanik"1. The final superposition of a living and a dead Cat was for him as a ridiculous

${ }_{1}^{1}$ E. Schrödinger "Die Gegenwärtige Situation in der Quantenmechanik” Die Naturwiss., 23 (1935), 807-812, 824-828, 844-849; E. Schrödinger, "Die Gegenwärtige Situazion in der Quantenmechanik", Sonderdruck aus die Naturwissenschaften, 1935. 23 Jahrg, Heft 48, 484-489. Heft 49, pp. 490-495. Heft 50, pp. 496-500. In: Gesammelte Abhandlungen, Band 3, Beitraege zur Quantentheorie, Verlag der Oesterreichischen Akademie der Wissenschaften, Wien 1984; Engl. translation by Trimmer (1983), The Present Situation in Quantum Mechanics: Proceedings of the American Philosophical Society, 124, pp. 323-338.

How to cite this paper: D'Agostino, S. (2015). A Good Chance for the Cat's Life: Erwin Schroedinger's New Statistics and a Second Quantization Theory of Quantum. Advances in Historical Studies, 4, 1-7. 
case (German: burleske Faelle), because he interpreted it as a result of the Copenhagen philosophy of QM². There was enough agreement among scholars to support the thesis that much of S.'s criticism was addressed against Bohr's and Heisenberg's views, and the Copenhagen interpretation of QM. But it was not commonly remarked that his criticism also concerned Einstein's interpretation of a QM measurement as the result of a classically statistical approach to the measurement problem. He believed that according to the New Statistics, a contradiction was inherent in keeping intact the particle concept and conceding that the particle possessed no individuality. The contradiction became a relevant topic in his discussion of the many particles problem, and of the second quantization approach to QM. I underline that the Cat episode is to be analyzed by taking account of S.'s earlier and later contribution to a philosophy of QM.

I show that the Cat episode has an interesting epistemological significance. Its roots are in S.'s earlier 1926 contributions to the rarified Gas theory. His positions in 1935 GS represented the basis for his next 1950 reflections on the meaning of the New Statistics. In my study, I also underline the importance of S.'s 1944 and 1952 contributions to his philosophy of a wavelike conception of QM. I close my study with a short comment on the epistemological significance of the Cat episode.

\section{The "Burlesque" Result of a Half Alive and Half Death Cat}

The Cat's ambiguous situation is presented and commented mainly at Sections 4, 5 and 10 of the first Part of Schrödinger famous 1935 essay, "Die Gegenwärtige Situation in der Quantenmechanik” (here to fore shortened GS). S.'s approach to the Cat's dramatic situation, usually described in the technical literature as a psi-wave collapse, is by S. narrated in a quite different form. In fact, S. presented it at Section 5 by asking the question: “Are the variables really blurred” [German: “Sind the Variablen wirklich verwischen?”]. S.'s answer is decisively negative.

The reason for refusing the "blurred" situation approach is presented in the same passage, by the following argument: the psi function is a mathematically good substitute as long as the "blurring" is confined to the atomic scale. But "serious misgivings arise if one notices that the term blurring is simply wrong for macroscopically tangible and visible thinks”. S.'s view of the transformation of a microscopic into a macroscopic state can be thus summarized: if the initial situation of the radioactive particles is interpreted as one into which two discharged and not discharged states are undistinguished (German: Indistinct), its transformation in a clear distinction between a live and a dead Cat, following the opening of the Cat's cage, is a clearly contradictory process. The "blurred state" should then be responsible for the probabilistic macroscopic catastrophe of the poor Cat's death or alive. The Cat's destiny following the blurred state is by S. regarded as a "ridicule case" ["burleske Faelle”], i.e., a case not worthy of serious consideration. To better underline his argument, S. continues by mentioning the difference between the snapshot of a cloud and that of an out of focus object: “... there is a difference between an indistinct or poorly focalized snapshot and that of a cloud”. Evidently the first photo can never be transformed in a set of clearly focused photos, just like as no distinction of live and dead Cat can follow by the initial blurred state. S.’s metaphorical expression “A fuzzy snapshot is not a cloud”, intends to underline that a cloud is not a superposition of neatly focused fogs, just like a fuzzy state can neither be interpreted as the superposition of clearly defined states. Out from metaphors, if the "state" of the microscopic radioactive system, responsible for a probabilistic macroscopic catastrophe and the poor Cat's death, is to be intended as a blurred state, it is contradictory to transform it in a macroscopic determined situation following the opening of the cat's cage. This explains why this result is by S. regarded as a "ridicule case” ["burleske Faelle”]. However, although the "ridicule case" ... prevents us from so naively accepting a "blurred model” for representing reality, "the blurred model in itself it would not embody anything unclear or contradictory". According to S., the "ridicule case” concerned the transformation of a microstate of electrons in a macrostate of a dead or a living Cat, but the

\footnotetext{
${ }^{2}$ S.'s passage on the Cat in Trimmer's English translation in: The Present Situation in Quantum Mechanics, quot., p. 323: One can even set up quite ridiculous cases. A cat is penned up in a steel chamber, along with the following device (which must be secured against direct interference by the cat): in a Geiger counter there is a tiny bit of radioactive substance, so small, that perhaps in the course of the hour one of the atoms decays, but also, with equal probability, perhaps none; if it happens, the counter tube discharges and through a relay releases a hammer which shatters a small flask of hydrocyanic acid. If one has left this entire system to itself for an hour, one would say that the cat still lives if meanwhile no atom has decayed. The psi-function of the entire system would express this by having in it the living and dead cat (pardon the expression) mixed or smeared out in equal parts. It is typical of these cases that an indeterminacy originally restricted to the atomic domain becomes transformed into macroscopic indeterminacy, which can then be resolved by direct observation. That prevents us from so naively accepting as valid a "blurred model" for representing reality. In itself it would not embody anything unclear or contradictory There is a difference between a shaky or out-of-focus photograph and a snapshot of clouds and fog banks.
} 
blurred microstate “... would not embody in itself anything unclear or contradictory”. Let us notice that S.’s underlined contradiction just arises from the transformation process from a micro into a macro state. S.'s thought experiment, certainly presents an imposing metaphorical interest, and the popular resonance of the Cat episode was certainly accrued by the biological incompatibility of the Cat's macrostates.

\section{An Analysis of S.'s Approach to the Cat Experiment}

As is known, the Cat episode offered to physicists and philosophers the occasion for many and variousinterpretations $^{3}$. The metaphorical power of the argument was certainly accrued by the biological antagonism between a Cat's life and death, but this feature would not justify for itself the one century long interest aroused by S.'s Cat among scientists and philosophers. There should exist some epistemological implications in the largely popular metaphorical situation: the Cat's unfortunate situation proves that the metaphor is not to be undervalued. S.'s criticism of the micro-macro transformation of the Cat episode also teaches to us that in QM a macro state das not consist into an assemblage of microstates, and, conversely that a microstate is not a very shortened Cat's macrostate. I believe that the peculiar aspect of S.'s argument, it is to be appreciated as a key for a better analysis of the nature and role of transformations in a quantum theory ${ }^{4}$.

A great interest is therefore to be attributed to S.'s 1935 correspondence with Einstein ${ }^{5}$. It is known that the exchange of letters interfered with S.'s positions on the Cat. S.'s letters to Einstein dated July 13, August 19, and October 4, and thus preceded his November 29 GS. His ideas on a rejection of the Copenhagen thoughts were initially in a substantial agreement with Einstein's EPR. In his August 19final correspondence however, he harshly criticized E.'s statistical interpretation of $\mathrm{QM}^{6}$. This less known detail presents one of the first instances in which Schrödinger ideas differentiated from Einstein's positions. He never accepted to again agree with Einstein's interpretation of $\mathrm{QM}^{7}$. Other circumstances influenced S.'s composition. The first part of GS concerning the Cat was published on Heft 29 of Sonderdruch of Naturwissenschaften and the other two parts were published later on the N. 49 and 50 Hetften. Since Sections 5 and 10 of the first part are related with Section 4, the first part of GS has a consistency of its own. It seems probable that it was put together only after the August 19 letter with E., and was added to the original manuscript sent to the editor Berliner ${ }^{8}$. This circumstance might contribute to explain the peculiar features of the Cat story. I argue in fact, that the Cat has its roots in the whole context of Schrödinger's contributions to a wave like theory of $\mathrm{QM}^{9}$. His interest to the problem of the relationship between particles and waves dates at least from his 1926 interpretation of the rarified Bose gas and the new statistics. In fact, in his 1926 contribution to E.'s theory of the Rarified Gas, he had remarked that the core of the passage from “Boltzmann's natural” to Bose's statistics was a conceptual interchange between the multiplicity of the energy particles and the multiplicity of their energy states. But this passage, he added, was equivalent to apply the De Broglie-Einstein wave-like theory, according to which a particle in motion is no less than a "crest of foam" on top of a radiation. It seems reasonable to argue that the passage of Bose's statistics might have impressed S. as a special case of a more general theory: en ensemble of particles is not composed of individuals but it is just a distribution of their energy states, Let us remark that the last conception can be considered as a forerunner of his 1950 generalization of the interchange between particles and state. His 1926 approach was in fact rightly considered as "his coming of age" in confront of what he had later on to achieve. In his initial approach in fact, he was not as yet aware that it represented an example of the fecundity of a New Statistics approach, the revolutionary progress that had to wait until his 1950 discovery.

In 1928, S.'s decision to abandon the electrodynamics interpretation of the psi-wave, made public in a paper ${ }^{10}$,

\footnotetext{
${ }^{3}$ Jammer (1966) The Conceptual Development of Quantum Mechanics, New York, Mc Grow-Hill Book Co., p. 271.

${ }^{4}$ Let us note that the first Chapter of A. P. Dirac's nowadays classical work, The Principles of QM, just concerns a Transformation Theory of QM.

${ }^{5}$ Fine (1986) The Shaky Game, Eintein's Realism and the Quantum Theory. The Univ. of Chicago Press, Chapter Five, pp. 64-85.

${ }^{6}$ Ibid, p. 79. In his August 19 letter S. criticized E.'s interpretation of the result of quantum measurement under the aspect of a classical statistical experiment.

${ }^{7}$ The negative conclusion of his epistolary exchanges with Einstein is dramatically expressed in S.'s final comment on Einstein's view at 4 October 1935: “Das gehetnichts” (Fine, quot., Chapter Five, pp. 64-85. On p. 79.

${ }^{8}$ This hypothesis was supported by Arthur Fine in his detailed analysis of the Einstein-Schroedinger collaboration on the Cat episode (Fine, A., quot., ChapterFive, pp. 64-85). I find that some aspects of GS might be taken as a support to Fine's hypothesis.

${ }^{9}$ S. D’Agostino, "Il gatto di S. in una lettura originale del celebre saggio”. Physis.Rivista Internazionale di Storia sella Scienza, Vol. XLVI. NS Fasc. 1-2, pp. 235-252.

${ }^{10}$ Schrödinger (1929) NeueWege in der Physik, Elektrotechnische Zeitschr, 50, 15-16.
} 
was justified by the impossibility of a space-time description of micro processes, because of Heisenberg's Relations (IR). He believed that IR "have altered our conception of a world-image and what we should intend as its description" ". A fundamental turning point was reached in his 1931 essay and concerned the theoretical relevance of his New Statistics approach. By extending his 1926 already mentioned interpretation of the rarified Bose Gas, he thought that the lack of individuality in the atomic world was an aspect of a more general crisis in the ontology of classical atomism, an inner failure of the classical objectification of distinct particles, and of the related Boltzmann Statistics distribution. The concept of indistinct particles, was a tenet for the foundation for his New Statistics. It afforded a major incentive for a radical innovation in his conception of physical systems and of their states. Let us remark that this innovation was one of the topic discussed in his 1935 problem of the Cat's state.

\section{The Dublin Papers in the 1940's and 50'. The Second Quantization Approach to the Cat}

In 1933 S. and Dirac received the Nobel Prize for "their discovery of new forms of atomic theories and their applications”. Ten years later, Schrödinger's and his wife's lives were upset by the sudden occurrence of their escape from Nazi Austria. In 1944, he accepted a welcome refuge and an opportunity for further research at the recently established Dublin Institute for Theoretical Physics. He therein continued to be interested in a radical innovation in his views of a wave -like QT. His radical criticism of the Copenhagen philosophy is evidenced in the 1950 article, What is an elementary particle ${ }^{12}$. The 1930' "many dimensional treatment" of a plurality of particles was in fact now superseded by the so-called second quantization, which is mathematically equivalent to uniting into a three-dimensional formulation the cases $\mathrm{N}=0,1,2,3, \cdots$ (to infinity) particles of the many-dimensional treatment. The new approach was marvelously consistent with the relevance given to his New Statistics:

This highly ingenious device includes the so-called new-statistics... It is the only precise formulation of the views now held, and the one that is always used. What is very significant in our present context is that one cannot avoid leaving indeterminate the number of the particles dealt with. It is thus obvious that they are not individuals.

And he added: "It may indeed well be that no individual entity can be conceived which would answer the requirements for the adequate picture stated above” ${ }^{\not 1}$. This remark underlines the old statistics contradiction due to keeping intact the particle concept and, at the same time, conceding that the particle possesses no individuality. His conception of a restricted notion of the physical particles identity, is splendidly illustrated by a new metaphor, offered in one section of the 1950 paper. In a semi-popular mood he presented an example of an analogical model or", as he named it, a "simile" of the fact that different statistical approaches are related to different ways of selecting physical system (the objects) and their states (the properties) ${ }^{14}$.

In the simile ${ }^{15}$, three schoolboys deserve rewards and the available awards are of three different kind: 1) Two portraits of Newton and of Shakespeare. 2) Two shilling-pieces, each piece an indivisible quantity). 3) Two vacancies in the school foot-ball team. According to the kinds of awards, different distributions of the awards among the boys are possible ${ }^{16}$.

\footnotetext{
${ }^{11}$ Schroedinger, Ueber Indeterminismus in der Physik. Istdie Naturwissenshaft Milieubedingt? A lecture to the Congress of the Society for the Teaching of Philosophy, June 16, 1931, Bartht, Leiptzig 1932.

${ }^{12}$ Schrödinger (1950) What is an Elementary Particle? In: Endevour 9, 109-116. Also: E. Schrödinger, Gesammelte Abhandlungen, Verlag der Oesterrreichischen Akademieder Wisseschaften, Wien 1984, Band 4. pp. 456-467.

${ }^{13}$ Ibid, p. 461.

${ }^{14}$ Notice the analogy with the early case of his 1926 essay on the rarified Gas.

${ }^{15}$ S., "What is an elementary particle?", S. Ges. Abhanl, cit. p. 461.

${ }^{16}$ I summarized S.'s distribution by the following scheme:
}

\begin{tabular}{ccccc}
\hline Awards & Distinguishable awards & Cumulativeness. & Number of distr. & Statistics \\
\hline 1) Two portraits & Yes & No & 9 & Boltzmann \\
2) Two shillings & No & Yes & 6 & Böse-Einst. \\
3) Two vacancies & No & No & 3 & Fermi-Dirac \\
\hline
\end{tabular}

${ }^{*}$ Cumulative = Cumulativeness; Disting. $=$ Distinguishability; Distr. = Distributions. 
Le us notice that the common-sense distribution criteria is somehow reversed, because boys represent the states of the system and allocation of awards represent the objects. In fact, a foot-ball team vacancy offered to the boy Dick means that the particle "vacancy" takes on the "state Dick". The identity of the three boys is thus somehow restricted, reproducing in the simile Schrödinger's conception of a restricted notion of the physical objects' identity. Notice that the non-cumulatively of awards, in case 1 and 3, illustrates Pauli's exclusion principle, whereas cumulativeness of case 2 corresponds to a second quantization approach to the Cat. Another Schrödinger's simile conveys the following metaphorical meaning: if, according to classical physics, one wishes to represent the staff's positions (roles) in a bank as corresponding to the energy-states of particles and the staff's members themselves as the classical particles, then Schrödinger's new conception compels one to reverse the correspondence, i.e. to alike the staff's positions (roles) to the new-statistical objects and the staff members themselves to the energy states ${ }^{17}$. The interest of the 1951 essay is also related to an original approach to the fundamental QM feature of a non-classical superposition of states. In a semi-popular mood, Schrödinger presented to his readers a striking example of the fact that different statistics require different (from the usual ones) approaches to the superposition problem. Notice that the Cat episode could now be regarded as an early case of particles state superposition.

The problem of a many particles wave-like theory took again S.'s attention in his 1952 essay, "Are there Quantum Jumps" ${ }^{\text {18 }}$. In Section 6, he remarked that in thought experiments we sometimes assume that the psi function is dealing with just one electron, but this is a conceptual model which does not apply to the $\mathrm{N}$ particles dealt with in a real experiment. The contradiction becomes evident though the following remark: it is commonly accepted that the number $\mathrm{N}$ entails $\geq \mathrm{N}$ fluctuations. The $\mathrm{N}$ values of the wave function are therefore spread over a region of $\geq \mathrm{N}$ magnitude. The fact that in a thought experiment we imagine to deal with just one electron and a definite wave function has the ridiculous consequence of a contradiction. Notice the clear reference to his approach to the Cat situation. And he added: If we wish to account for the beautiful experiment of G. P. Thomson on the interference patterns of De Broglie waves, we "sorely need" to consider them as physical waves, not just the expression of our psi wave QM lack of knowledge. The spherical wave effect of the emitted particle, a topic in his GS, is thus again presented as a proof of the difficulties inhering in the theoretical model, because of the impossibility of experimenting with just one particle. He hinted thus at a conflict between classical theory and experiments. Let us remark that this was a fundamental theme of his Dublin years philosophy ${ }^{19}$.

\section{Meeting with De Broglie at the Henry Poincare Institute in Paris}

Problems concerning the distinction of particles and the equations multidimensionality were again met by him in 1953, in a paper ${ }^{20}$ written on the occasion of the commemoration of De Broglie's sixtieth birthday, held at the Henry Poinc are Institute, in Paris. Schrödinger initiates his discourse by remarking that he himself and De Broglie were shocked and disappointed when “... [they] learnt that a sort of transcendental, almost psychical interpretation of the wave phenomenon has been put forward". To this interpretation-evidently the Copenhagen interpretation of QM-Schrödinger opposed his and De Broglie's own interpretation via the wave function. Responding to the accuse that his former wave-like interpretation seemed deceptive and, after all, too naive, Schrödinger accepted to reconsider it in the light of two new points which have since arisen: 1) not distinguishable particles, following the New-Statistics; 2) second-quantization procedures. He added that they are intimately connected... " $\{$ and $\}$ have not turned up suddenly. Their roots lie far back, but their bearing was only very gradually recognized”"21. As regards 2), he stated that “... if a particle is not a permanent entity, quantization of the De Broglie Waves around a nucleus welds into one comprehensive scheme all the 3n-dimensional represen-

\footnotetext{
${ }^{17}$ Let us remind that Schrödinger made a similar remark concerning the interpretation of a gas state in the kinetic theory.

${ }^{18}$ Schrödinger (1952) Are there Quantum Jumps? School of Theoretical Physics, Dublin Institute for Advanced Studies.

${ }^{19}$ D’Agostino (1992) “Continuity and Completeness in Physical Theory: S.’s return to the wave interpretation of QM in the 1950’s”, pp. 339-360. In Erwin Schrödinger. Philosophy and the Birth of QM, edited by Bitbol M. and Darrigol O. Edition Frontières, Gif-sur Yvette Cedex-France.

${ }^{20}$ Schroedinger (1953) La signification de la Mecanique Ondulatoire. In Louis De Broglie, Physicien at Penseur, Collection Dirigée par André George Albin Michael, pp. 2-32.

${ }^{21}$ Ibid, p. 28.
} 
tations that I had proposed for the n-body problem”22.

In 1952, Schrödinger abandoned his researches on Generalized Field Theories with a pessimistic outlook on their future. In his Dublin residence he had just begun a series of lectures and publications-some of them at a non-professional level — on philosophy and history of science. They were collected and published in 1951 under the title: Science and Humanism ${ }^{23}$. In 1954 he published Nature and the Greeks, followed in 1957 by Science Theory and Man, and, in I958, by Mind and Matter. Let us remark that his 1956 What is Life? and Other Scientific Lectures, a work very rightly considered the beginning of the future Biophysics, was born in period of S.'s strong philosophical interests. What he proposed was a renewed view of Wave Mechanics, in which his early theories on the physical meaning of the psi-function and of the New Statics were now re-examined in the light of his new conception of physical theory.

\section{Concluding Remarks}

In this paper I support the thesis that S.'s 1930 and 1950 positions represent a development of themes that can be traced to his 1926 contributions to rarified Gas Theory and to his enduring epistemological interests. The relevance of his epistemological interests on his approach to his celebrated Cat is one of the themes of my paper. Though, as to my present knowledge, my thesis has been almost ignored in S.'s historical literature, his epistemological contributions in the Fifties, have been often interpreted ${ }^{24}$ as a manifestation of an absence of a theoretical engagement, his unwillingness to work out a full-fledged physical theory. My explanation for this absence is different. I think that an overall judgment on the nature and scope of Schrödinger's scientific contributions in the fifties should take into account that he intended to keep open the chances for a future continuous theory of microphysics, which could oppose the then triumphant Copenhagen philosophy. In this sense, his work represented the fulfillment of what he considered his intellectual mission. As an initial approach, at least, he thought that it was important to make clear the general epistemological context and the foundations of his theory and to develop a radical criticism of the Copenhagen philosophy. But he was aware of the amount of efforts and devotion required in order to produce significant innovations within the body of the predominant paradigm of his contemporary physics. This work represented in his views only rarely a one-man task. In 1952, somehow confessing his impotence in front of the enormity of this task, he complained: "I beg to plead that I am at the moment groping for my way almost single handed, as against a host of clever people doing their best along the recognized lines of thought” ${ }^{\prime 25}$. In an historical perspective, one may judge his intellectual isolation from the world center of scientific enterprise, in the usually more fruitful period of his scientist's life, as a consequence of the events in his life in the pre-war and war years, mainly as a consequence of his opposition to the Nazi power. But one should also admit that his isolation started earlier, around 1926, when he began to oppose the triumphant Copenhagen interpretation of QM. Was this isolation the consequence of his opposition to those unquestionable answers which nature supposedly dispenses to its scientific adepts, or was it just "largely a sociological accident"? ${ }^{26}$ The fact that modern physicists abandoned Schrödinger's wave theory of QM, and, more then else,

\footnotetext{
${ }^{22}$ As a modern expression of Schrödinger’s 1926 psi equation for the case of $\mathrm{N}$ identical particles one has:$$
i \frac{h}{2 \pi} \frac{\partial \psi(1,2, \cdots N ; t)}{\partial t}=H(1,2, \cdots N) \psi(1,2, \cdots N ; t)
$$

The solution for the psi is not factorable, and represent mathematically the physical situation of the actual fuzzy behavior of Schrödinger psi for his Cat in a firstly quantized theory. As the metaphor of the Cat illustrates, in a first quantization theory, the superposition of two incompatible electron micro states cannot be transformed into two compatible biological Cat's macro states.

Quite differently, the description given for the factorial $\Psi$ for an ensemble of $\mathrm{N}$ particles in the second quantization approach is factorable. In fact, in the case of Bose particles the expression for $\Psi$ is:

$$
\Psi_{N_{1} N_{2} \cdots N}=\sqrt{N_{I} ! N_{2} ! \cdots / N ! \Sigma} \phi_{p_{1}}\left(\chi_{1}\right) \phi_{p_{2}}\left(\chi_{2}\right) \cdots \phi_{p_{n}}\left(\chi_{n}\right)
$$

Ni number of particles in the state $\phi_{1}$. Ni may, of course, bee zero. Here p1, p2, $\cdots, \mathrm{pN}$, are the ordinal numbers of the states in which the individual particles are, and the sum is taken over all permutations of those suffixes p1, p2, $\cdots, \mathrm{pN}$, which are different from the particles' properties permutations.

Let us note that the $\Psi$ given by second quantization is factorable as a product of psi functions of the particles states. It expresses a known formula for a compound probability. In the case of two states it provides distinct Cat alive or Cat dead states.

${ }^{23}$ Schroedinger, E., Science and Humanism. Physics in our Times, Cambridge Un. Press, 1051.

${ }^{24}$ Wessels (1983) Ervin Schroedinger and the descriptive tradition. In R. Arris et al., Eds., Springs of scientific Creativity, Minneapolis, 254-278.

Bertotti (1987) Presentazione. In: Erwin Schrödinger, L’immagine del Mondo, Borinhieri, p. 8.

${ }^{25}$ Schrödinger, E. In: Louis De Broglie, Physicien at Penseur, quot. p. 28.

${ }^{26}$ Pickering (1984) Constructing Quarks. A sociological History of Particle Physics. Edinburgh University Press.
} 
its philosophical premises, whereas they continue using his operational equations to follow their computations, might represent an answer to the questions above. Historians may find answers to such questions through a detailed study of the available sources and a search for unknown ones. A deeper analysis of his own and of other contemporary theories, indicated by him in his papers, seems indispensable for a reconstruction of a wave-like theory of microphysics in accordance with Schrödinger's views. This remark suggests that the work of the historical profession cannot be substituted by other parenthetical or provisional approaches. Physicists can also contribute to an improvement of the analysis of Schrödinger's theories by deepening some aspects of his contributions. In the last decade, Schrödinger's philosophy on a wave-like theory of microphysics returned in fact to the forefront of physicists' interest due to complications in the Standard Model of microphysics.

The study of S.'s thought would certainly contribute to an improvement in our understanding of the physical and epistemological conceptions of one of the most outstanding European Scientist-philosophers of our time.

\section{Acknowledgements}

I wish to thank my friends and colleagues Stefania Borgo and Lucio Sibilia for their insightful comments to my paper.

\section{References}

Bertotti, B. (1987). Presentazione. Erwin Schrödinger. L'immagine del Mondo (p. 8). Borinhieri.

D’Agostino, S. (1992). Continuity and Completeness in Physical Theory: S.'s Return to the Wave Interpretation of QM in the 1950's. In M. Bitbol, \& O. Darrigol (Eds.), Erwin Schrödinger. Philosophy and the Birth of QM (pp. 339-360). Gif-sur Yvette Cedex: Edition Frontières.

Fine, A. (1986). The Shaky Game, Eintein's Realism and the Quantum Theory (Chapter Five, pp. 64-85). Chicago: The Univ. of Chicago Press.

Jammer, M. (1966). The Conceptual Development of Quantum Mechanics (p. 271). New York: Mc Grow-Hill Book Co.

Pickering, A. (1984). Constructing Quarks. A Sociological History of Particle Physics. Edinburgh: Edinburgh University Press.

Schrödinger, E. (1929). NeueWege in der Physik. Elektrotechnische Zeitschr, 50, 15-16.

Schrödinger, E. (1950). What is an Elementary Particle? Endevour, 9, 109-116.

Schrödinger, E. (1952). Are There Quantum Jumps? School of Theoretical Physics, Dublin Institute for Advanced Studies.

Schroedinger, E. (1953). La signification de la Mecanique Ondulatoire. In Louis De Broglie, Physicien at Penseur (pp. 2-32). Collection Dirigée par André George Albin Michael.

Trimmer, J. D. (1983). The Present Situation in Quantum Mechanics. Proceedings of the American Philosophical Society, 124, 323-338.

Wessels, L. (1983). Ervin Schroedinger and the Descriptive Tradition. In R. Arris et al. (Eds.), Springs of Scientific Creativity (pp. 254-278). Minneapolis. 